\title{
Original Article \\ Garlic Increases Antioxidant Levels in Diabetic and Hypertensive Rats Determined by a Modified Peroxidase Method
}

\author{
Hana Drobiova, Martha Thomson, Khaled Al-Qattan, Riitta Peltonen-Shalaby, \\ Zainab Al-Amin, and Muslim Ali
}

Department of Biological Sciences, Faculty of Science, Kuwait University, P.O. Box 5969, 13060-Safat, Kuwait

Correspondence should be addressed to Martha Thomson, mmtkuwait2003@hotmail.com

Received 12 August 2008; Accepted 16 January 2009

Copyright (C) 2011 Hana Drobiova et al. This is an open access article distributed under the Creative Commons Attribution License, which permits unrestricted use, distribution, and reproduction in any medium, provided the original work is properly cited.

\begin{abstract}
Oxidative damage by free radicals has been implicated in the pathogenesis of vascular disease in diabetes and hypertension. In the present study, the total antioxidant status in diabetic and hypertensive rats before and after treatment with garlic (Allium sativum) was determined. The total serum antioxidants were measured by a modified method reported earlier by Miller and coworkers. The reproducibility of the assay was confirmed by determining standard curves for the known antioxidants: trolox (a stable analog of vitamin E), glutathione and vitamin $\mathrm{C}$ with interassay correlation coefficients $\left(R^{2}, n=10\right.$ in triplicate) of $0.9984,0.9768$ and 0.987, respectively, confirming the reliability and reproducibility of the assay. This assay was then used to determine total serum antioxidant levels of streptozotocin-induced diabetic rats and two-kidney one-clip hypertensive rats both before and after 3 weeks of treatment with an aqueous extract of garlic $(500 \mathrm{mg} / \mathrm{kg}$ IP daily). The serum antioxidant levels of rats after 3 weeks of treatment were significantly higher $(P<.001)$ than the pretreatment levels in both diabetic and hypertensive rats. The increased serum antioxidant levels were paralleled by a decrease in serum glucose in the garlic-treated diabetic rats and lowered systolic blood pressure in the garlic-treated hypertensive rats. We conclude from our study that (i) total antioxidants can be measured by a simple, reproducible, reliable assay and (ii) the total antioxidant status can be significantly improved by treatment with garlic.
\end{abstract}

\section{Introduction}

Oxidative stress, an excessive production of reactive oxygen species (ROS) above the body's antioxidant capacity, has been implicated in the development of many pathophysiological conditions including hypertension, diabetes, atherosclerosis and cancer, as well as the process of aging [1-6]. ROS are normal products of cellular metabolism which are usually removed by endogenous antioxidants [7]. However, it has become increasingly clear that overproduction of ROS can lead to a damaging cycle of lipid peroxidation, depletion of natural antioxidants such as glutathione, perturbation of nitric oxide production and disruption of normal cellular metabolism [8]. These changes have been shown to cause damage to cell membranes and in particular can cause endothelial dysfunction [9].

Due to the importance of changes in the oxidant to antioxidant ratio in relation to the development of pathological conditions, the measure of antioxidant capacity of serum has increasingly become used as an index of the antioxidant status of the system. Markers of oxidative stress that are commonly measured include: endogenous antioxidants such as glutathione, cysteine and antioxidant vitamins; antioxidant enzymes such as glutathione peroxidase, myeloperoxidase and superoxide dismutase; $\mathrm{F}_{2}$-isoprostanes and malondialdenyde as products of lipid peroxidation; and end-products of protein oxidation such as nitrotyrosine $[8$, 10]. In addition, a number of in vitro spectrophotometric assays have been developed to assess antioxidant capacity including the quantitation of Trolox equivalent antioxidant capacity $[8,11]$. However, many of these assays are laborious, involve the use of difficult and unclear methodologies and are expensive. In a recent review, Dalle-Donne and coworkers have discussed the measurement and reliability of various biomarkers in human disease [10].

Recent reports have suggested that diabetic complications and hypertension have a common etiology involving oxidative stress and endothelial damage $[9,12,13]$. In fact, 
the earliest pathological changes in diabetic complications have been reported to be hemodynamic in nature [1416]. Thus, the assessment of oxidative stress in both diabetes and hypertension has become crucial as an index of pathology of these diseases. In fact, many reports have emphasized the common link between ROS levels and severity of these conditions $[17,18]$. In addition, antioxidants have been shown to modulate oxidative stress-induced changes in hypertension, atherosclerosis and diabetes [19, 20].

Due to the increasing worldwide prevalence and financial burden of chronic diseases such as diabetes and hypertension, it has become increasingly important to find pharmacological remedies to alleviate the symptoms and complications of these conditions. In particular the use of natural remedies such as garlic has become popular as both preventative and treatment alternatives. Garlic has been extensively studied and has been shown to have a number of medicinal properties including antithrombotic [21-26], antibiotic [2729], hypolipidemic [30, 31], hypocholesteremic [30, 32], hypoglycemic [33-39] and hypotensive [30, 40] activities. Various garlic preparations and components have also been shown to have antioxidant activity including the ability to lower ROS in vivo [31, 41, 42].

In recent years, we have been studying the beneficial effects of aqueous extracts of garlic in the treatment of chronic diseases such as diabetes and hypertension [21, 23$25,30,43,44]$. As part of this work, we found it necessary to develop a method for measurement of oxidative stress. Measurement of Trolox equivalent antioxidant capacity units (TEAC) was developed by Miller, Rice-Evans and coworkers as a method for measurement of antioxidant capacity [45-47]. This is an electron-transfer method that exploits the peroxidase activity of methemoglobin combined with its interaction with a phenothiazine compound [azinobis(3-ethylbenzothiazoline-6-sulfonic acid), ABTS] to form a radical cation intermediate $\left(\mathrm{ABTS}^{\circ+}\right)$. The concentration of antioxidants giving the same percentage change of absorbance of $\mathrm{ABTS}^{*+}$ as that of $1 \mathrm{mM}$ Trolox is regarded as a TEAC [48]. However, description of this method lacked critical details necessary to its routine use. Therefore, in the present study, the assay was modified for routine measurement of antioxidant capacity of serum and was applied to the assessment of antioxidant levels in diabetic and hypertensive rat models treated with garlic.

\section{Materials and Methods}

2.1. Materials. Streptozotocin, myoglobin (equine) and 2,2'azinobis-(3-ethylbenzothiazoline-6-sulphonic acid) diammonium salt (ABTS) were purchased from Sigma Chemical Co. (St Louis, MO, USA). Trolox (6-hydroxy-2,5,7,8tetramethylchronam-2-carboxylic acid) was purchased from Aldrich Chemical Co. (Milwaukee, WI, USA). Vitamin C, reduced glutathione and hydrogen peroxide were obtained from Fluka (Buchs, Switzerland). Glucose oxidase kits were obtained from Waco Pure Chemicals Co., Ltd (Osaka, Japan). All other reagents were of analytical grade.
2.2. Antioxidant Assay. Total antioxidant levels were measured by a modified Rice-Evans method [45-47] which is based on the inhibition by antioxidants of the absorbance of the radical cation $\mathrm{ABTS}^{\bullet+}$. The spectrophotometric method derives from the observation that when ABTS is incubated with a peroxidase (metmyoglobin) and hydrogen peroxide, the relatively long-lived radical cation, $\mathrm{ABTS}^{\circ+}$, is formed. ABTS $^{\bullet+}$ has absorption maxima at 650,734 and $820 \mathrm{~nm}$. In the presence of antioxidants, the absorbance (at $734 \mathrm{~nm}$ ) of this radical cation is quenched to an extent and on a time scale dependent on the antioxidant capacity of the material under investigation.

Metmyoglobin (MetMb) was prepared by mixing myoglobin with an oxidizing agent $\left[\mathrm{K}_{3} \mathrm{Fe}\left(\mathrm{CN}_{6}\right)\right]$ and then repurified on a G-25 column before use as described by Miller and Rice-Evans [47]. The concentration of myoglobin in the column eluate was calculated using the extinction coefficients of met-, oxy- and ferryl-myoglobin at 490, 560 and $580 \mathrm{~nm}$ using the following equation:

$$
[\mathrm{MetMb}](\mu \mathrm{M})=146 A_{490}-108 A_{560}+2.1 A_{580} .
$$

Purified myoglobin was stored in small aliquots at $-20^{\circ} \mathrm{C}$ until use.

The current modification of the assay included changes in the specificity of timing of the assay including a $3 \mathrm{~min}$ preincubation that are critical to the reproducibility of the assay (Table 1). Three known antioxidants, vitamin C, trolox (a stable analog of vitamin E) and glutathione, were used to assess the reproducibility of the assay. The results are calculated and expressed as TEAC.

2.3. Preparation of Garlic Extract. Aqueous garlic extract was prepared from locally available garlic cloves. The garlic cloves were peeled on crushed ice and $50 \mathrm{~g}$ of garlic was cut into small pieces and homogenized in $75 \mathrm{~mL}$ of cold, sterile $0.9 \%$ $\mathrm{NaCl}$ in the presence of some crushed ice. The homogenization was carried out in a blender at high speed using six 2-min bursts for a total of $12 \mathrm{~min}$. The homogenized mixture was filtered three times through cheesecloth. The filtrate was centrifuged at $2000 \mathrm{RCF}$ for $10 \mathrm{~min}$ and the clear supernatant was made up to $100 \mathrm{~mL}$ with normal saline. The concentration of this garlic preparation was considered to be $500 \mathrm{mg} / \mathrm{mL}$ on the basis of weight of the starting material $(50 \mathrm{~g} / 100 \mathrm{~mL})$.

2.4. Diabetes Induction and Sample Collection. Male SpragueDawley rats weighing 250-280 g and maintained on a normal diet and filtered tap water ad libitum were used in the study. All animal manipulations were conducted according to the ethical guidelines outlined in the Guide for Care and Use of Laboratory Animals [49]. For baseline data, blood was drawn from all animals by cardiac puncture under ether anesthesia and allowed to clot. Immediately, the clotted blood was centrifuged at $2000 \mathrm{RCF}$ for $30 \mathrm{~min}$. The serum was separated and stored at $-80^{\circ} \mathrm{C}$ for later analysis.

The animals were randomly divided into a normal group (eight rats) and a streptozotocin (STZ)-treated group (20 rats). The STZ-treated rats were injected with $60 \mathrm{mg}$ 
TABle 1: Protocol for the antioxidant assay.

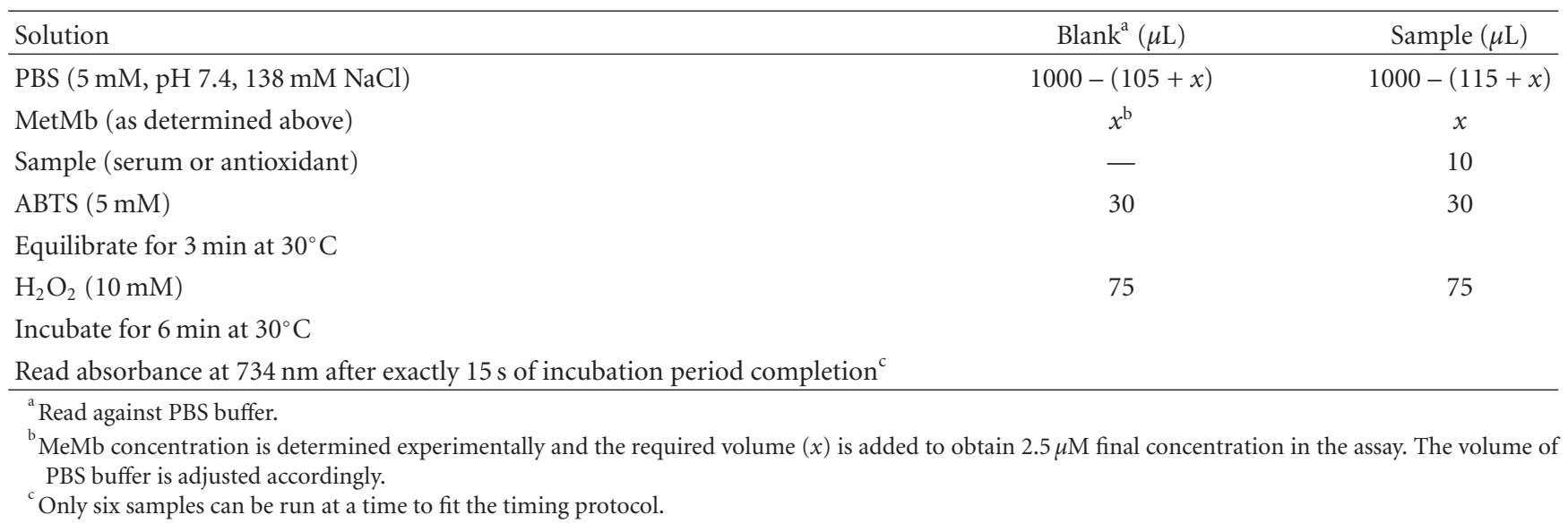

steptozotocin/ $\mathrm{kg}$ body weight intraperitoneally (IP) in a volume of $0.5 \mathrm{~mL}$ saline according to the method of Axler [50] following an overnight fast. After a period of 3 days, blood was drawn from the STZ-treated animals by cardiac puncture, and serum was prepared and stored for later analysis as described above. Serum glucose levels were determined immediately and the STZ-treated rats determined to be diabetic due to a high serum glucose level (>350 mg/dL) were randomly divided into two groups containing eight animals each: Group 1, the diabetic control group, was injected IP daily with saline for the treatment period; and Group 2, the garlic-treated group, was injected IP daily with $500 \mathrm{mg} / \mathrm{kg}$ of the garlic extract.

After a period of 3 weeks, the rats were sacrificed under sodium pentobarbitone anaesthesia according to the guidelines for euthanasia in the Guide for Care and Use of Laboratory Animals [49]. Before sacrifice, blood was collected, processed and stored at $-80^{\circ} \mathrm{C}$ for analysis within 1 week as described above.

2.5. Induction of Hypertension and Assessment. Ten male Sprague-Dawley rats with initial body weight of $50 \mathrm{~g}$ were operated on to induce a two-kidney, one-clip (2K-1C) model of hypertension as described earlier [51]. After 1 week, hypertension was confirmed by blood pressure measurement using the tail-cuff method. The hypertensive rats were then divided into two groups: five of the rats were treated daily with $500 \mathrm{mg} / \mathrm{kg}$ garlic IP for a period of 3 weeks and the remaining five rats served as control and were given saline IP for 3 weeks. Blood pressure was also measured at the end of the treatment period by the tail-cuff method. Blood was drawn by cardiac puncture before and after 3 weeks of treatment as described above and total antioxidant levels were determined.

2.6. Assays. Serum glucose was quantitated spectrophotometrically by the glucose oxidase method using kits from Waco, (Japan). TEAC levels (mM) were determined as described above. Systolic blood pressure was measured using the tail-cuff technique (Harvard Apparatus, England).
2.7. Statistical Analysis. The experimental data are expressed as mean \pm SEM. Statistical analysis was performed using Minitab Statistical Software (Version 7, State College, PA, USA). Data was analyzed using the two-sample $T$-test and a level of $P<.05$ is considered to be significant.

\section{Results}

3.1. Assessment of the Assay. The reproducibility of the antioxidant assay was assessed by determination of the inhibitory effects of three known antioxidants, vitamin C, glutathione and trolox (a stable analog of vitamin $\mathrm{E}$ ). The standard curve for each antioxidant was performed 8-10 times in triplicate. The standard curves in Figures 1(a)1(c) indicate that for all three known antioxidants good reproducibility within the concentration ranges was obtained using the assay. The inter-assay correlation coefficients $\left(R^{2}\right)$ were $0.9984,0.9768$ and 0.987 for trolox, glutathione and vitamin $\mathrm{C}$, respectively, confirming the reliability and reproducibility of the assay.

3.2. Effects in Diabetic Rats. Induction of a diabetic state with STZ led to marked hyperglycemia within 3 days of injection (Table 1). This hyperglycemic state was accompanied by significantly decreased antioxidant levels (about 40\% reduction) both 3 days and 3 weeks after STZ-injection (Figure 2). In contrast, after 3 weeks, the diabetic rats given $500 \mathrm{mg} / \mathrm{kg}$ garlic daily recovered antioxidant activity reaching levels in excess of those observed in normal rats. This increase in serum antioxidant levels was paralleled by a decrease in serum glucose levels as the garlic-treated diabetic rats returned to an euglycemic state (Table 2 ).

3.3. Effects in Hypertensive Rats. As shown in Figure 3, 2K$1 \mathrm{C}$ hypertensive rats (systolic $\mathrm{BP}=200 \pm 17 \mathrm{~mm}$ ) exhibited significantly decreased total antioxidant levels compared to normotensive rats (about 66\% reduction). After 3 weeks of garlic treatment, the antioxidant levels in the hypertensive rats increased to about two-third of the normal levels, while the antioxidant levels in the hypertensive control rats 


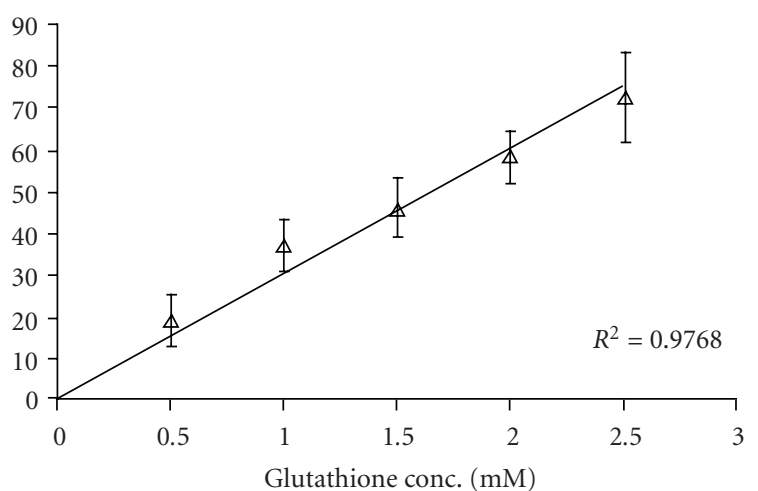

(a)

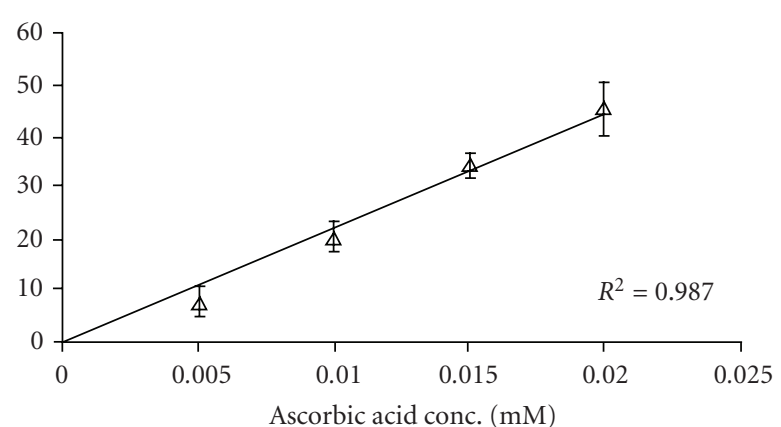

(b)

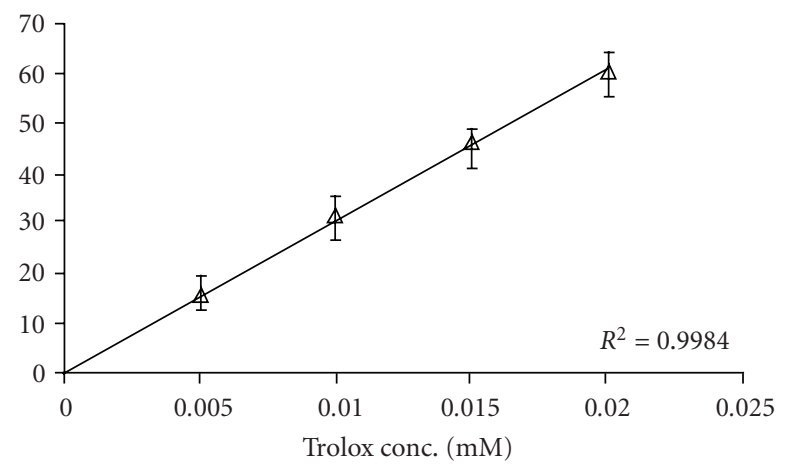

(c)

Figure 1: Standard curves for inhibition of $\mathrm{ABTS}^{+}$absorbance at $734 \mathrm{~nm}$ with (a) glutathione, (b) ascorbic acid (vitamin C) and (c) trolox. Glutathione concentrations were between 0.5 and $2.5 \mathrm{mM}$. Ascorbic acid concentrations were between 0.005 and $0.02 \mathrm{mM}$. Trolox concentrations were between 0.005 and $0.02 \mathrm{mM}$. All assays were done in triplicate.

TABLE 2: Serum glucose concentration in different animal groups.

\begin{tabular}{lcc}
\hline & Initial serum glucose $(\mathrm{mg} / \mathrm{dL})$ & Final serum glucose $(\mathrm{mg} / \mathrm{dL})$ \\
\hline Normal $^{\mathrm{a}}$ (saline treated) & - & $129 \pm 9$ \\
Diabetic control $^{\mathrm{a}}$ (Saline treated) & $400 \pm 9$ & $431 \pm 13^{\mathrm{b}}$ \\
Garlic-treated diabetic $^{\mathrm{a}}(500 \mathrm{mg} / \mathrm{kg}$ garlic) & $400 \pm 9^{\mathrm{c}}$ & $237 \pm 8^{\mathrm{c}}$ \\
\hline
\end{tabular}

\footnotetext{
${ }^{a} n=10$ for all groups.

${ }^{\mathrm{b}}$ Significally different compared to normal $(P<.05)$.

${ }^{\mathrm{c}}$ Significally different compared to initial and final diabetic controls $(P<.001)$.
}

continued to decrease (about $20 \%$ of the normotensive level) during the 3-week experimental period. This increase in antioxidant levels occurred simultaneously with a $50 \%$ decrease in blood pressure (to $100 \pm 22 \mathrm{~mm}$ systolic) in the $2 \mathrm{~K}-1 \mathrm{C}$ hypertensive rats.

\section{Discussion}

During the last decade, it has become increasingly evident that many chronic diseases are accompanied by increased levels of oxidative stress exacerbated by decreased antioxidant levels [10, 17, 52-54]. These observations have precipitated much interest in study of the correlations between oxidative stress, antioxidant potential and development of chronic diseases in both humans and animal models. Of particular interest are the correlations between oxidative stress and development of diabetes and hypertension $[4,5$, 9, 55-57].

With the surge in research in this area, the development of simple, reliable and consistent methods for quantitation of oxidative stress has become imperative. In particular, assays for serum antioxidant levels have been developed including measurement of total peroxy radical trapping as described by Wayner et al. [58] and Rice-Evans and Miller [45-47]. Whereas the original peroxy radical trapping parameter assay of Wayner and coworkers [57] required an oxygen electrode endpoint, Rice-Evans and Miller developed this assay to use a spectrophotometric endpoint [45]. As described above, this assay involves utilization of the peroxidase activity of 


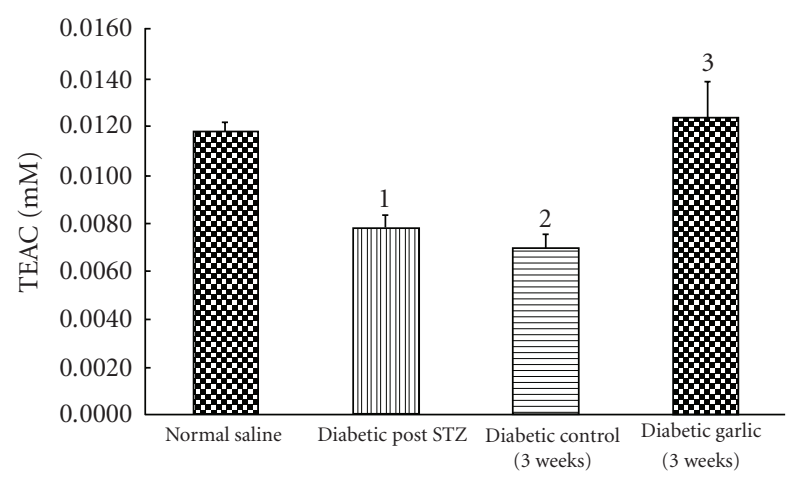

Figure 2: Antioxidant levels in diabetic rats. Normal, untreated (control) diabetic and garlic-treated diabetic (diabetic garlic) rats were assessed for antioxidant status (TEAC) 1 week after STZ-injection (Post-STZ) and 3 weeks after treatment. (1) Total antioxidants in normal rats are significantly hgher than in diabetic rats 3 days after induction of diabetes with STZ $(P<.05)$. (2) Total antioxidants in normal rats are significantly higher than in diabetic control rats after 3 weeks $(P<.05)$. (3) Total antioxidants in diabetic control rats are significantly lower than in garlic-treated diabetic rats after 3 weeks of garlic treatment $(P<.05)$.

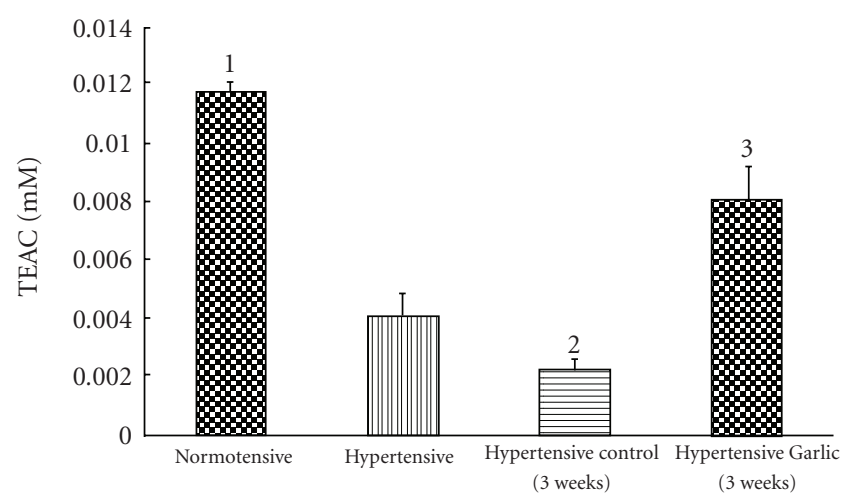

FIGURE 3: Antioxidant levels in hypertensive rats. Normal, untreated (control) hypertensive and garlic-treated hypertensive rats were assessed for antioxidant status (TEAC) before and 3 weeks after treatment. (1) Total antioxidants in normotensive rats are significantly higher than in hypertensive rats $(P<.05)$. (2) Total antioxidants in hypertensive rats at time zero are significantly higher than hypertensive rats after 3 weeks $(P<.05)$. (3) Total antioxidants in untreated hypertensive rats are significantly lower than garlictreated hypertensive rats after 3 weeks of garlic treatment $(P<.05)$.

methemoglobin, generation of the radical cation, $\mathrm{ABTS}^{\bullet+}$, and quantitation of the quenching of this radical cation by antioxidants. Results are expressed in Trolox equivalent antioxidant capacity using the vitamin E analog, Trolox, as the reference antioxidant.

In the present study, we have modified the Rice-Evans and Miller assay $[46,47]$ to include more precise timing parameters including a 3-min pre-incubation, a 6-min reaction time followed by absorbance measurement exactly $15 \mathrm{~s}$ after stopping the inhibition reaction. These timing parameters have allowed the assay to be conducted with the use of a $30^{\circ} \mathrm{C}$ water bath and a basic spectrophotometer. The
Rice-Evans and Miller manual assay [46] required the use of a temperature-controlled cuvette holder for continuous measurement of absorbance. Thus, in the current modification, the assay involves pre-incubation of all reagents except $\mathrm{H}_{2} \mathrm{O}_{2}$ for $3 \mathrm{~min}$ at $30^{\circ} \mathrm{C}$, addition of $\mathrm{H}_{2} \mathrm{O}_{2}$ at $15 \mathrm{~s}$ intervals followed by $6 \mathrm{~min}$ incubation at $30^{\circ} \mathrm{C}$. The absorbance was then read at exactly $15 \mathrm{~s}$ intervals such that all assays were incubated for exactly $6 \mathrm{~min}$. These timing parameters allowed six assays to be carried out simultaneously. The 6 min reaction time is in agreement with that of van den Berg et al. [59] and Re and coworkers [60], who also modified the assay to eliminate the use of methemoglobin. In our hands, the modifications described have allowed the routine use of this assay for assessment of serum antioxidant levels. Standard curves for three known antioxidants (gluathione, vitamin C and Trolox) indicate that the assay is reproducible and stable (Figures 1(a)-1(c)).

There has been considerable focus in recent years on the connection between oxidative stress and endothelial dysfunction leading to the development of chronic conditions such as diabetes and hypertension $[5,9,13,56,61]$. Endothelial dysfunction is correlated with the development of coronary heart disease and peripheral vascular resistance which are the leading causes of morbidity and mortality in diabetes mellitus $[18,62]$. It has become increasingly evident that the development of hyperglycemia followed by oxidative stress is the underlying cause of the chronic complications of diabetes. Therefore, the prevention or alleviation of oxidative stress in diabetes should positively benefit the diabetic patient.

Similarly, the development of hypertension has been linked to impairment in the antioxidant defense system $[5,56]$. Similar to diabetes, hypertension, in particular renovascular hypertension, has been characterized by increased oxidative stress and endothelial dysfunction [63]. Therefore, numerous studies have focused on the correlation between serum antioxidant levels and hypertension as well as the alleviation of this chronic condition through increased consumption of antioxidants and antioxidant-rich foods [64, 65 .

Our results show that treatment of STZ-induced diabetic rats with a raw garlic extract alleviates both hyperglycemia and oxidative stress in these animals. These observations are in agreement with those of Anwar and Meki [31] who treated STZ-induced diabetic rats with garlic oil. In addition, recently Zalejska-Fiolka and coworkers [66] reported that garlic-fed rats also fed oxidized oil had improved antioxidant status, less lipid peroxidation and fewer atherosclerotic changes than in oxidized oil fed controls. Similarly, Souza and coworkers [67] have reported that $N$-acetylcysteine (NAC), a water-soluble compound found in garlic extracts, improved high-sucrose diet-induced obesity in rats. NAC treatment resulted in improved glucose tolerance and lipid profile, as well as decreased LDL-oxidation and serum oxidative stress. Therefore, several studies have suggested that garlic most likely exacerbates diabetes in animal models through improved antioxidant status.

Therefore, we have shown the applicability of the TEAC assay to the assessment of antioxidant status of diabetic 
rats. It should be noted that the method described here measures only water-soluble antioxidants. Therefore, the results reported here do not include fat-soluble antioxidant levels which also may be affected by garlic treatment.

In previous studies, we have reported that the $2 \mathrm{~K}-1 \mathrm{C}$ rat model is characterized by the development of renal hypertension accompanied by increased expression of two isoforms of the $\mathrm{Na} / \mathrm{H}$ exchanger (NHE-1 and-3) in both kidneys and increased serum levels of $\mathrm{PGE}_{2}$ and $\mathrm{TXB}_{2}$ $[43,68]$. Treatment of these rats with an aqueous extract of garlic lowered blood pressure and NHE-1 expression in the unclipped kidney [43]. In the present study, we have observed that the development of renal hypertension in the $2 \mathrm{~K}-1 \mathrm{C}$ rat is accompanied by decreased levels of serum antioxidants. The increased blood pressure and oxidative stress were alleviated by administration of an aqueous extract of garlic. These results are in agreement with our earlier observation that the hypotensive effect of garlic is partially mediated through the nitric oxide pathway in the $2 \mathrm{~K}-1 \mathrm{C}$ rat model confirming the usefulness of garlic in decreasing oxidative stress and hypertension [44].

\section{Summary}

From this study, the following conclusions can be stated. A reliable and reproducible assay for determination of total antioxidant levels in serum has been developed and applied. Treatment of diabetic rats with garlic resulted in significantly increased antioxidant and lowered glucose levels compared to untreated diabetic animals. Treatment of $2 \mathrm{~K}$ $1 \mathrm{C}$ hypertensive rats with garlic increased the total level of antioxidants to about two-third of the normal level. Garlictreated $2 \mathrm{~K}-1 \mathrm{C}$ hypertensive rats had significantly higher total antioxidant levels and lower systolic blood pressure than untreated $2 \mathrm{~K}-1 \mathrm{C}$ hypertensive rats.

\section{Acknowledgment}

This work was supported by the College of Graduate Studies, Kuwait University for which the authors express gratitude.

\section{References}

[1] B. Halliwell, "Free radicals, antioxidants, and human disease: curiosity, cause, or consequence?" The Lancet, vol. 344, no. 8924, pp. 721-724, 1994.

[2] D. Giugliano, A. Ceriello, and G. Paolisso, "Diabetes mellitus, hypertension, and cardiovascular disease: Which role for oxidative stress?" Metabolism, vol. 44, no. 3, pp. 363-368, 1995.

[3] T. Finkel and N. J. Holbrook, "Oxidants, oxidative stress and the biology of ageing," Nature, vol. 408, no. 6809, pp. 239-247, 2000.

[4] N. F. Wiernsperger, "Oxidative stress: the special case of diabetes," BioFactors, vol. 19, no. 1-2, pp. 11-18, 2003.

[5] R. M. Touyz, "Reactive oxygen species, vascular oxidative stress, and redox signaling in hypertension: what is the clinical significance?” Hypertension, vol. 44, no. 3, pp. 248-252, 2004.

[6] C. Chinopoulos and V. Adam-Vizi, "Calcium, mitochondria and oxidative stress in neuronal pathology: novel aspects of an enduring theme," FEBS Journal, vol. 273, no. 3, pp. 433-450, 2006.

[7] H. Sies, Oxidative Stress: Oxidants and Antioxidants, Academic Press, New York, NY, USA, 1991.

[8] M. H. Shishehbor and S. L. Hazen, "Antioxidant studies need a change of direction," Cleveland Clinic Journal of Medicine, vol. 71, no. 4, pp. 285-288, 2004.

[9] U. Bayraktutan, "Free radicals, diabetes and endothelial dysfunction," Diabetes, Obesity and Metabolism, vol. 4, no. 4, pp. 224-238, 2002.

[10] I. Dalle-Donne, R. Rossi, R. Colombo, D. Giustarini, and A. Milzani, "Biomarkers of oxidative damage in human disease," Clinical Chemistry, vol. 52, no. 4, pp. 601-623, 2006.

[11] G. Cao and R. L. Prior, "Comparison of different analytical methods for assessing total antioxidant capacity of human serum," Clinical Chemistry, vol. 44, no. 6, pp. 1309-1315, 1998.

[12] H. A. R. Hadi and J. A. Al Suwaidi, "Endothelial dysfunction in diabetes mellitus," Vascular Health and Risk Management, vol. 3, no. 6, pp. 853-876, 2007.

[13] M. R. Nicolls, K. Haskins, and S. C. Flores, "Oxidant stress, immune dysregulation, and vascular function in type I diabetes," Antioxidants and Redox Signaling, vol. 9, no. 7, pp. 879-889, 2007.

[14] M. Brownlee, "Biochemistry and molecular cell biology of diabetic complications," Nature, vol. 414, no. 6865, pp. 813820, 2001.

[15] M. J. Sheetz and G. L. King, "Molecular understanding of hyperglycemia's adverse effects for diabetic complications," JAMA, vol. 288, no. 20, pp. 2579-2588, 2002.

[16] R. Engerman, J. M. B. Bloodworth Jr., and S. Nelson, "Relationship of microvascular disease in diabetes to metabolic control," Diabetes, vol. 26, no. 8, pp. 760-769, 1977.

[17] R. Halliwell and J. M. C. Gutteridge, Free Radicals in Biology and Medicine, Oxford University Press, New York, NY, USA, 1999.

[18] A. Ceriello, "Oxidative stress and diabetes-associated complications," Endocrine Practice, vol. 12, no. 1, pp. 60-62, 2006.

[19] A. Ceriello, "New insights on oxidative stress and diabetic complications may lead to a "causal" antioxidant therapy," Diabetes Care, vol. 26, no. 5, pp. 1589-1596, 2003.

[20] S. Vasdev, V. D. Gill, and P. K. Singal, "Modulation of oxidative stress-induced changes in hypertension and atherosclerosis by antioxidants," Experimental and Clinical Cardiology, vol. 11, no. 3, pp. 206-216, 2006.

[21] M. Ali, M. Thomson, M. A. Alnaqeeb, J. M. Al-Hassan, S. H. Khater, and S. A. Gomes, "Antithrombotic activity of garlic: its inhibition of the synthesis of thromboxane- $\mathrm{TXB}_{2}$ during infusion of arachidonic acid and collagen in rabbits," Prostaglandins Leukot Essent Fatty Acids, vol. 41, pp. 95-99, 1990.

[22] H. Kiesewetter, F. Jung, G. Pindur, E. M. Jung, C. Mrowietz, and E. Wenzel, "Effect of garlic on thrombocyte aggregation, microcirculation, and other risk factors," International Journal of Clinical Pharmacology Therapy and Toxicology, vol. 29, no. 4, pp. 151-155, 1991.

[23] M. Ali and M. Thomson, "Consumption of a garlic clove a day could be beneficial in preventing thrombosis," Prostaglandins Leukot Essent Fatty Acids, vol. 53, pp. 211-212, 1995.

[24] T. Bordia, N. Mohammed, M. Thomson, and M. Ali, "An evaluation of garlic and onion as antithrombotic agents," Prostaglandins Leukotrienes and Essential Fatty Acids, vol. 54, no. 3, pp. 183-186, 1996.

[25] M. Thomson, T. Mustafa, and M. Ali, “Thromboxane- $B_{2}$ levels in serum of rabbits receiving a single intravenous dose of 
aqueous extract of garlic and onion," Prostaglandins Leukot Essent Fatty Acids, vol. 63, pp. 217-221, 2000.

[26] M. Steiner and W. Li, "Aged garlic extract, a modulator of cardiovascular risk factors: a dose-finding study on the effects of AGE on platelet functions," Journal of Nutrition, vol. 131, no. 3, pp. 980S-984S, 2001.

[27] L. P. Rees, S. F. Minney, N. T. Plummer, J. H. Slater, and D. A. Skyrme, "A quantitative assessment of the antimicrobial activity of garlic (Allium sativum)," World Journal of Microbiology \& Biotechnology, vol. 9, no. 3, pp. 303-307, 1993.

[28] H. Yoshida, N. Iwata, H. Karsuzaki et al., "Antimicrobial activity of a compound isolated from an oil-macerated garlic extract," Bioscience, Biotechnology, and Biochemistry, vol. 62, pp. 1014-1017, 1998.

[29] I. M. Bakri and C. W. I. Douglas, "Inhibitory effect of garlic extract on oral bacteria," Archives of Oral Biology, vol. 50, no. 7, pp. 645-651, 2005.

[30] M. Ali, K. K. Al-Qattan, F. Al-Enezi, R. M. A. Khanafer, and T. Mustafa, "Effect of allicin from garlic powder on serum lipids and blood pressure in rats fed with a high cholesterol diet," Prostaglandins Leukotrienes and Essential Fatty Acids, vol. 62, no. 4, pp. 253-259, 2000.

[31] M. M. Anwar and A.-R. M. A. Meki, "Oxidative stress in streptozotocin-induced diabetic rats: effects of garlic oil and melatonin," Comparative Biochemistry and Physiology-Part A, vol. 135, no. 4, pp. 539-547, 2003.

[32] C. Stevinson, M. H. Pittler, and E. Ernst, "Garlic for treating hypercholesterolemia: a meta-analysis of randomize clinical trials," Annals of Internal Medicine, vol. 133, no. 6, pp. 420I31, 2000.

[33] C. G. Sheela and K. T. Augusti, "Antidiabetic effects of S-allyl cysteine sulphoxide isolated from garlic Allium sativum Linn," Indian Journal of Experimental Biology, vol. 30, no. 6, pp. 523526, 1992.

[34] C. G. Sheela, K. Kumud, and K. T. Augusti, "Anti-diabetic effects of onion and garlic sulfoxide amino acids in rats," Planta Medica, vol. 61, no. 4, pp. 356-357, 1995.

[35] K. T. Augusti, "Therapeutic values of onion (Allium cepa L.) and garlic (Allium sativum L.)," Indian Journal of Experimental Biology, vol. 34, no. 7, pp. 634-640, 1996.

[36] S. Kasuga, M. Ushijima, N. Morihara, Y. Itakura, and Y. Nakata, "Effect of aged garlic extract (AGE) on hyperglycemia induced by immobilization stress in mice," Nippon Yakurigaku Zasshi, vol. 1999, pp. 191-197, 1999.

[37] G. S. Eisenbarth and B. L. Kotzin, "Enumerating autoreactive T cells in peripheral blood: a big step in diabetes prediction," The Journal of Clinical Investigation, vol. 111, no. 2, pp. 179181, 2003.

[38] G. A. Jelodar, M. Maleki, M. H. Motadayen, and S. Sirus, "Effect of fenugreek, onion and garlic on blood glucose and histopathology of pancreas of alloxan-induced diabetic rats," Indian Journal of Medical Sciences, vol. 59, pp. 64-69, 2005.

[39] C.-T. Liu, H. Hse, C.-K. Lii, P.-S. Chen, and L.-Y. Sheen, "Effects of garlic oil and diallyl trisulfide on glycemic control in diabetic rats," European Journal of Pharmacology, vol. 516, no. 2, pp. 165-173, 2005.

[40] S. K. Banerjee and S. K. Maulik, "Effect of garlic on cardiovascular disorders: a review," Nutrition Journal, vol. 1, article 1, pp. 1-14, 2002.

[41] S. Mizuguchi, S. Takemura, Y. Minamiyama et al., "Sallyl cysteine attenuated $\mathrm{CCl}_{4}$-induced oxidative stress and pulmonary fibrosis in rats," BioFactors, vol. 26, no. 1, pp. 8192, 2006.
[42] A. Avci, T. Atli, I. B. Ergüder et al., "Effects of garlic consumption on plasma and erythrocyte antioxidant parameters in elderly subjects," Gerontology, vol. 54, no. 3, pp. 173-176, 2008.

[43] K. K. Al-Qattan, I. Khan, M. A. Alnaqeeb, and M. Ali, "Mechanism of garlic (Allium sativum) induced reduction of hypertension in $2 \mathrm{~K}-1 \mathrm{C}$ rats: a possible mediation of $\mathrm{Na} / \mathrm{H}$ exchanger isoform-1," Prostaglandins Leukotrienes and Essential Fatty Acids, vol. 69, no. 4, pp. 217-222, 2003.

[44] K. K. Al-Qattan, M. Thomson, S. Al-Mutawa'a, D. Al-Hajeri, H. Drobiova, and M. Ali, "Nitric oxide mediates the bloodpressure lowering effect of garlic in the rat two-kidney, oneclip model of hypertension," Journal of Nutrition, vol. 136, no. 3, pp. 774S-776S, 2006.

[45] N. J. Miller, C. Rice-Evans, M. J. Davies, V. Gopinathan, and A. Milner, "A novel method for measuring antioxidant capacity and its application to monitoring the antioxidant status in premature neonates," Clinical Science, vol. 84, no. 4, pp. 407412, 1993.

[46] C. Rice-Evans and N. J. Miller, "Total antioxidant status in plasma and body fluids," Methods in Enzymology, vol. 234, pp. 279-293, 1994.

[47] N. J. Miller and C. A. Rice-Evans, "Spectrophotometric determination of antioxidant activity," Redox Report, vol. 2, no. 3, pp. 161-171, 1996.

[48] D. Huang, O. U. Boxin, and R. L. Prior, "The chemistry behind antioxidant capacity assays," Journal of Agricultural and Food Chemistry, vol. 53, no. 6, pp. 1841-1856, 2005.

[49] Veterinary Medical Care, in Guide for the Care and Use of Laboratory Animals, pp. 56-70, Institute for Laboratory Animal Research, American Academy of Sciences, Washington, DC, USA, 1996.

[50] D. A. Axler, "Stability of the diabetogenic activity of streptozotocin," IRCS Medical Science, vol. 10, no. 2, pp. 157-158, 1982.

[51] K. K. Al-Qattan, "Different levels of hypertension induce opposite diuretic behaviors from the nonclipped kidney in the rat two-kidney, one-clip model," Kidney and Blood Pressure Research, vol. 24, no. 1, pp. 44-51, 2001.

[52] B. Halliwell, "Role of free radicals in the neurodegenerative diseases: therapeutic implications for antioxidant treatment," Drugs and Aging, vol. 18, no. 9, pp. 685-716, 2001.

[53] R. Stocker and J. F. Keaney Jr., "Role of oxidative modifications in atherosclerosis," Physiological Reviews, vol. 84, no. 4, pp. 1381-1478, 2004.

[54] J. E. Klaunig and L. M. Kamendulis, "The role of oxidative stress in carcinogenesis," Annual Review of Pharmacology and Toxicology, vol. 44, pp. 239-267, 2004.

[55] J. W. Baynes, "Role of oxidative stress in development of complications in diabetes," Diabetes, vol. 40, no. 4, pp. 405412, 1991.

[56] R. M. Touyz, "Reactive oxygen species in vascular biology: role in arterial hypertension," Expert Review of Cardiovascular Therapy, vol. 1, no. 1, pp. 91-106, 2003.

[57] L. O. Medina, C. A. Veloso, E. de Abreu Borges et al., "Determination of the antioxidant status of plasma from type 2 diabetic patients," Diabetes Research and Clinical Practice, vol. 77, no. 2, pp. 193-197, 2007.

[58] D. D. M. Wayner, G. W. Burton, K. U. Ingold, L. R. C. Barclay, and S. J. Locke, "The relative contributions of vitamin E, urate, ascorbate and proteins to the total peroxyl radical-trapping antioxidant activity of human blood plasma," Biochimica et Biophysica Acta, vol. 925, pp. 409-419, 1987. 
[59] R. van den Berg, G. R. M. M. Haenen, H. van den Berg, and A. Bast, "Applicability of an improved Trolox equivalent antioxidant capacity (TEAC) assay for evaluation of antioxidant capacity measurements of mixtures," Food Chemistry, vol. 66, no. 4, pp. 511-517, 1999.

[60] R. Re, N. Pellegrini, A. Proteggente, A. Pannala, M. Yang, and C. Rice-Evans, "Antioxidant activity applying an improved ABTS radical cation decolorization assay," Free Radical Biology and Medicine, vol. 26, no. 9-10, pp. 1231-1237, 1999.

[61] M. A. Zimmerman and S. C. Flores, "Autoimmune-mediated oxidative stress and endothelial dysfunction: implications of accelerated vascular injury in type I diabetes," Journal of Surgical Research, vol. 155, no. 1, pp. 173-178, 2008.

[62] N. B. Ruderman, J. R. Williamson, and M. Brownlee, "Glucose and diabetic vascular disease," The FASEB Journal, vol. 6, no. 11, pp. 2905-2914, 1992.

[63] Y. Higashi, S. Sasaki, K. Nakagawa, H. Matsuura, T. Oshima, and K. Chayama, "Endothelial function and oxidative stress in renovascular hypertension," The New England Journal of Medicine, vol. 346, no. 25, pp. 1954-1962, 2002.

[64] V. Digiesi, M. Lenuzza, and G. Digiesi, "Prospects for the use of antioxidant therapy in hypertension," Annali Italiani $d i$ Medicina Interna, vol. 16, no. 2, pp. 93-100, 2001.

[65] B. Rodriguez-Iturbe, C.-D. Zhan, Y. Quiroz, R. K. Sindhu, and N. D. Vaziri, "Antioxidant-rich diet relieves hypertension and reduces renal immune infiltration in spontaneously hypertensive rats," Hypertension, vol. 41, no. 2, pp. 341-346, 2003.

[66] J. Zalejska-Fiolka, A. Kasperczyk, S. Kasperczyk, U. Blaszczyk, and E. Birkner, "Effect of garlic supplementation on erythrocytes antioxidant parameters, lipid peroxidation, and atherosclerotic plaque formation process in oxidized oil-fed rabbits," Biological Trace Element Research, vol. 120, pp. 195204, 2007.

[67] G. A. Souza, G. X. Ebnaid, F. R. F. Seiva et al., "Nacetylcysteine, an Allium plant compound improves highsucrose diet-induced obesity and related effects," EvidenceBased Complementary and Alternative Medicine, 2008, , doi:10.1093/ecam/nen070.

[68] I. Khan, K. K. Al-Qattan, M. A. Alnaqeeb, and M. Ali, "Altered expression of $\mathrm{Na}^{+} / \mathrm{H}^{+}$exchanger isoforms 1 and 3 in clipped and unclipped kidneys of a 2-kidney-1-clip Goldblatt model of hypertension," Nephron, vol. 92, no. 2, pp. 346-355, 2002. 


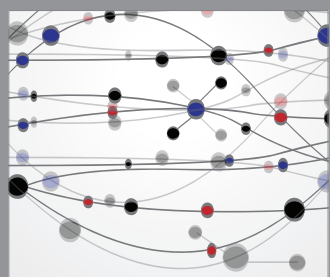

The Scientific World Journal
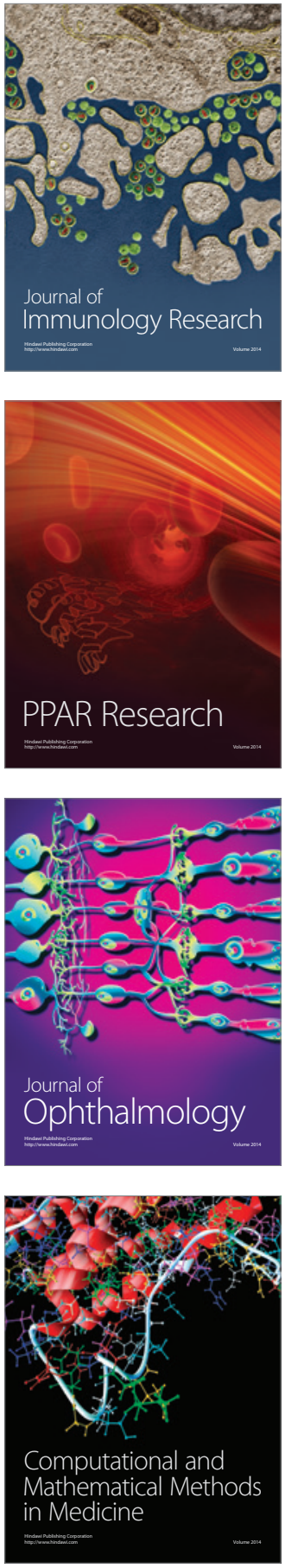

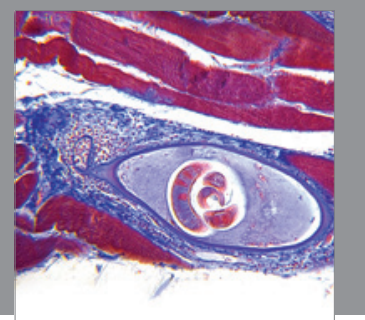

Gastroenterology

Research and Practice
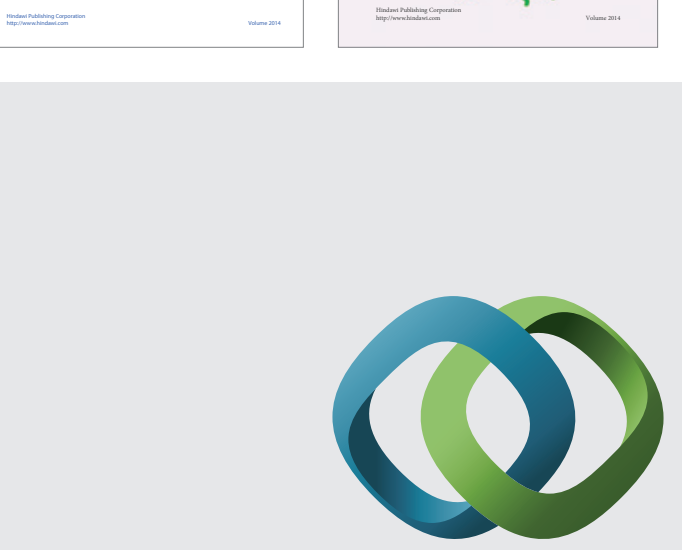

\section{Hindawi}

Submit your manuscripts at

http://www.hindawi.com
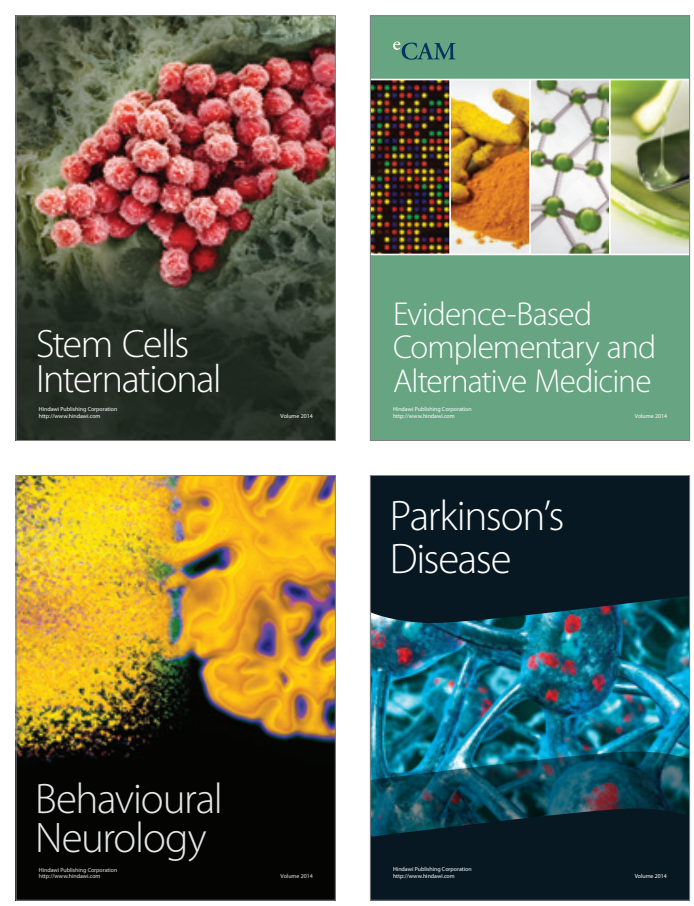

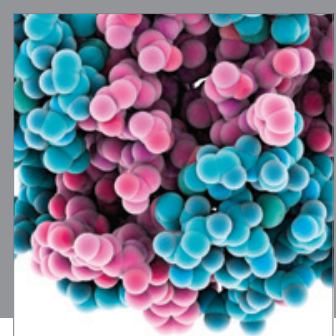

Journal of
Diabetes Research

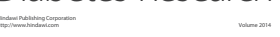

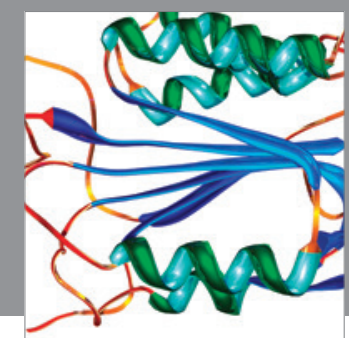

Disease Markers
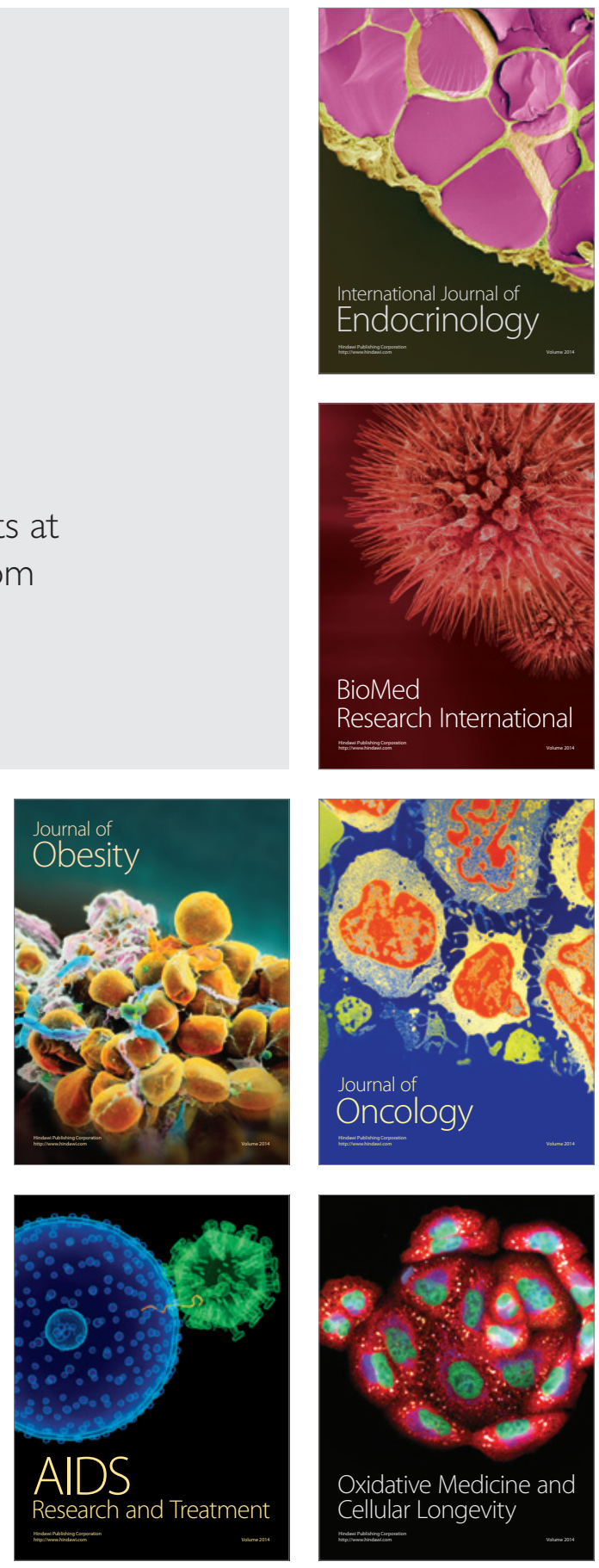